\title{
Laser Doppler imaging as a tool in the burn wound treatment protocol
}

\author{
Algirda Venclauskiene ${ }^{1}$, Algidas Basevicius², Ernest Zacharevskij ${ }^{3}$, Vytautas Vaicekauskas ${ }^{4}$, Rytis Rimdeika ${ }^{3}$, \\ Saulius Lukosevicius ${ }^{2}$ \\ ${ }^{1}$ Kaunas Clinics, Lithuanian University of Health Sciences, Kaunas, Lithuania \\ ${ }^{2}$ Department of Radiology, Kaunas Clinics, Lithuanian University of Health Sciences, Kaunas, Lithuania \\ ${ }^{3}$ Department of Plastic and Reconstructive Surgery, Kaunas Clinics, Lithuanian University of Health Sciences, Kaunas, Lithuania \\ ${ }^{4}$ Institute of Anatomy, Medical Academy, Lithuanian University of Health Sciences, Kaunas, Lithuania
}

Videosurgery Miniinv 2014; 9 (1): 24-30

DOI: $10.5114 /$ wiitm.2014.40273

\begin{abstract}
Introduction: The main treatment of burns is early excision of injured tissues.

Aim: To compare two different methods of examination of burned patients: clinical burn depth examination (CDE) and laser Doppler imaging (LDI).

Material and methods: A prospective randomized study of 57 burn patients treated in 2009-2011 was carried out. The burned patients were randomized into a CDE group and an LDI group. The CDE and LDI scan were performed $72 \mathrm{~h}$ after injury, with the second and third CDE and LDI scan on the $7^{\text {th }}$ and $14^{\text {th }}$ day after the burn. Age, sex, length of inpatient stay, cost of burn treatment, burn depth, cause and localization of the burns were analyzed between the two groups.

Results: Fifty-seven patients were treated during 2 years. Thirty-two patients were in the CDE group and 25 patients were in the LDI group. Most of the patients were male (45 male vs. 12 female, $p<0.001$ ). The age was similar between the males and the females (female: $46.4 \pm 16.9$ years vs. male: $46.3 \pm 12.5$ years; $p=0.11$ ). The mean length of stay in hospital was significantly higher in the CDE group $(47 \pm 34.4$ day vs. $25 \pm 10.8$ day; $p=0.005)$. The mean cost of treatment of burned patients was significantly higher in the CDE group.

Conclusions: The length of stay and cost of treatment of burn patients depends upon early diagnosis of the deep burns and well-timed surgical treatment of burn wounds.
\end{abstract}

Key words: laser Doppler imaging, perfusion velocity, burn depth measure.

\section{Introduction}

Trauma and burns are among the main sources of morbidity and mortality of the community in our days. Burns result in long inpatient stay and cause psychological problems [1, 2]. The main treatment of burns is early excision of injured tissues which reduce inpatient stay and decrease the costs of treat- ment [3-5]. There are many methods to assess burn wounds, but clinical examination of burn depth is still widely used $[6,7]$.

\section{Aim}

The aim of this clinical study was to compare two different methods of examination of burned pa-

\section{Address for correspondence}

Dr. Algirda Venclauskiene, Kaunas Clinics, Lithuanian University of Health Sciences, 2 Eiveniu St, LT50009 Kaunas, Lithuania, phone: +37068430783, e-mail: algirda.venclauskiene@yahoo.com 
tients - clinical burn depth examination (CDE) and laser Doppler imaging (LDI) - to estimate the impact of the methods on the length of patient's stay in hospital and the costs of burn treatment.

\section{Material and methods}

A prospective randomized study of 57 burn patients treated in the University Hospital of Kaunas, Department of Plastic and Reconstructive Surgery, in the period 2009-2011, was carried out. The clinical burn depth examination and LDI scan were performed for all burn patients who agreed to participate in the study. Inclusion criteria for the clinical study were patients burned within $72 \mathrm{~h}$, age 18-75 years, and agreement to participate in the study. Exclusion criteria were age $>75$ years, pregnant burned women, mental disease, lack of agreement to participate in the clinical study, and burned patients with a critical condition of health who were treated in the intensive care unit department. The patients were randomized $72 \mathrm{~h}$ after the burn. The burned patients were randomized into two groups: the CDE group and LDI group. Randomization of burned patients was done using envelopes with numbers inside them and a list on paper where numbers showed in which group the patients belonged. The CDE was performed by surgeons and the LDI scan was performed by a radiologist. A Moor LDLS laser Doppler line scanner (Moor LDLS Rapid laser Doppler blood flow imaging by Moor instruments) was used for the LDI scan. The distance between the burn wound and scanner was $15 \mathrm{~cm}$ and the scan angle was $90^{\circ}$.

The depth of the injured tissue was assessed during CDE and LDI scan. The depth of injured tissue was divided into superficial (I and IIA burn degree) and deep burns (IIB and III burn degree). The burn wound biopsy was performed by a surgeon in the same burn location to detect the correlation of find-

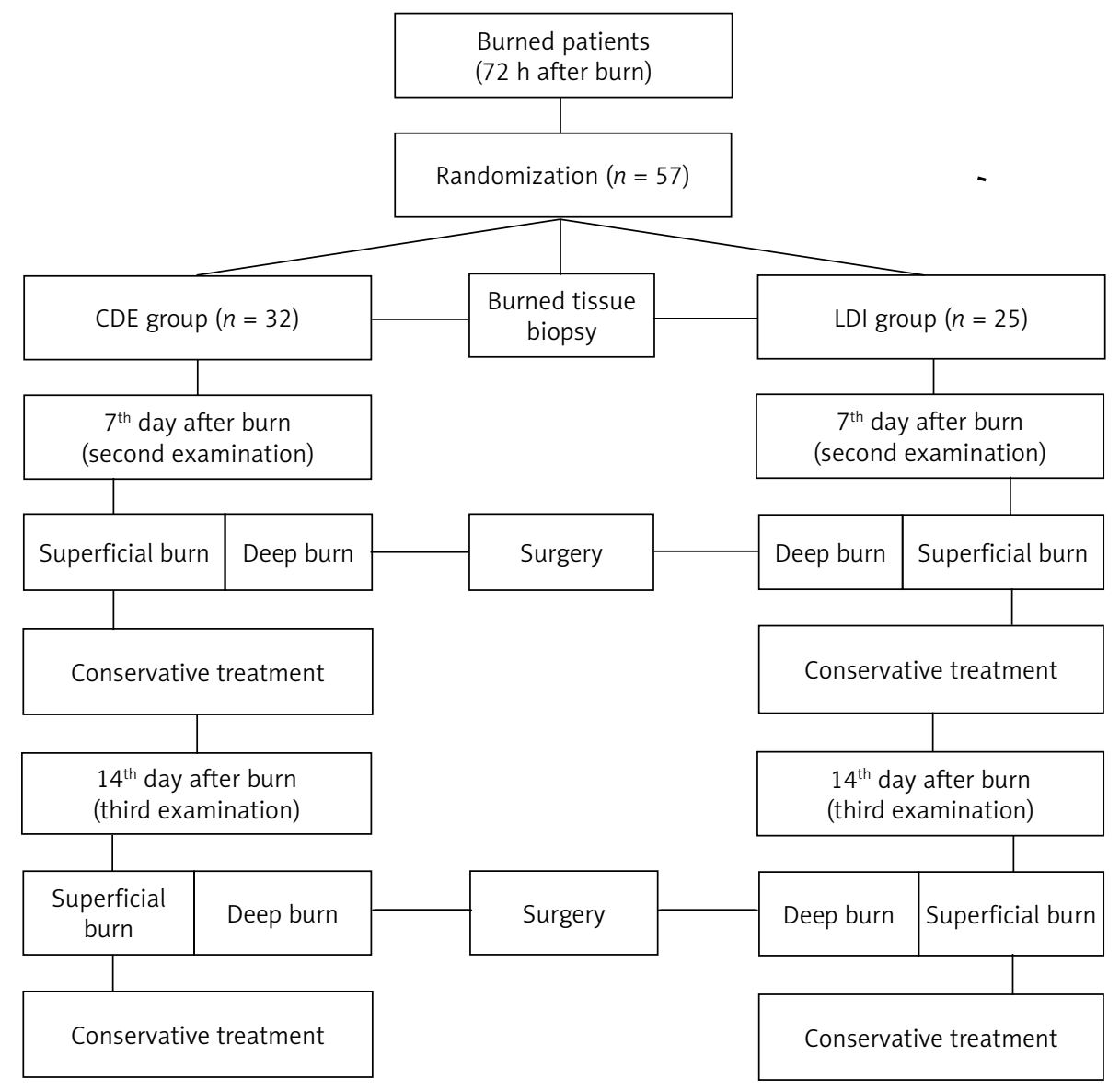

Figure 1. Scheme of patients' investigation 


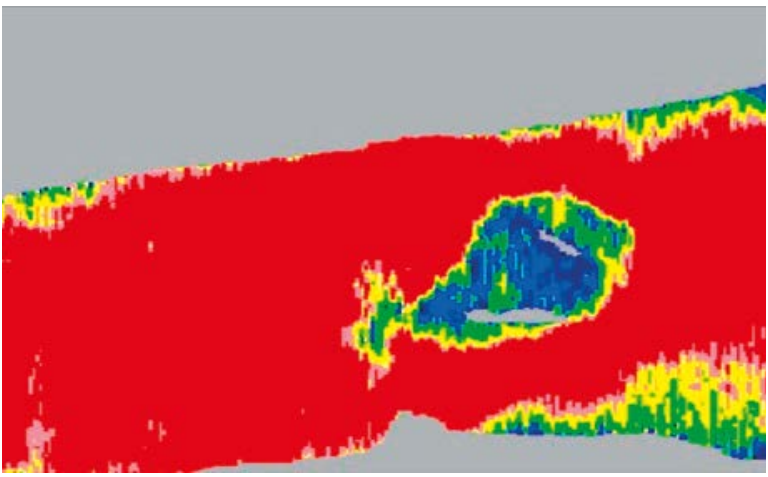

Photo 1. LDI scan (the arm). Burn tissue colors seen during LDI scan: blue and green color low perfusion of injury tissue (deep burn), yellow color - moderate perfusion (between superficial and deep burn), and red color - higher perfusion of injured tissue (superficial burn)

ings of CDE and LDI scan. The worst burn wound was elected during CDE and LDI scan.

The CDE, LDI scan and biopsy were performed $72 \mathrm{~h}$ after injury, with the second and third CDE and LDI scan on the $7^{\text {th }}$ and $14^{\text {th }}$ day after the burn. If during a clinical examination a surgeon decided to perform necrectomy of the burn tissue 7 days after injury, the patients' participation in the clinical study was finished. The third clinical examination (CDE group) was performed on the $14^{\text {th }}$ day after the burn. The patient's participation in the clinical study was finished when the surgeon decided to perform necrectomy or normal tissue epithelization was seen at that time. The indication for surgery of patients of the CDE group depended on the clinical surgeon's experience. The indication for surgery of patients of the LDI group depended on LDI scan parameters. A deep burn was assessed when perfusion velocity during the LDI scan on the $7^{\text {th }}$ and $14^{\text {th }}$ day after the burn was 0-250 PU (perfusion units). The patients underwent surgery. If perfusion velocity was > $250 \mathrm{PU}$ it was considered a superficial burn and patients were followed up and treated conservatively. The scheme of patient investigation is shown in Figure 1. The age, sex, length of inpatient stay, cost of burn treatment, burn depth, cause and location of the burns were analyzed between the two groups. The perfusion velocity of burn tissue was measured during the LDI scan, with results presented in perfusion units (PU). The LDI scan is shown in Photo 1.

The cost of burned wound treatment was calculated applying fixed valuation of service offering of the national health care system. Expense of treatment depended on the length of the patient's stay in hospital and operation or conservative treatment of the burn patient was done. The clinical study was approved by the Regional Ethic committee (No. BE 2-23; 11 Apr 2009).

Table I. General patient characteristics

\begin{tabular}{|c|c|c|c|c|}
\hline Parameter & & $\begin{array}{l}\text { CDE group } \\
(n=32)\end{array}$ & $\begin{array}{l}\text { LDI group } \\
(n=25)\end{array}$ & Value of $p$ \\
\hline \multirow[t]{2}{*}{ Sex } & Female & 6 & 6 & NS \\
\hline & Male & 26 & 19 & \\
\hline Age, mean $\pm S D$ & & $47.5 \pm 13.9$ & $44.8 \pm 12.7$ & NS \\
\hline Burn width (\%) & & $19.5 \pm 9.9$ & $18.2 \pm 14.5$ & NS \\
\hline \multirow[t]{4}{*}{ Cause of burn } & Flame & 27 & 16 & - \\
\hline & Hot water & 4 & 5 & - \\
\hline & Hot steam & - & 1 & - \\
\hline & Chemical sub. & 1 & 3 & - \\
\hline \multirow[t]{4}{*}{ Burn depth } & I & 1 & 3 & - \\
\hline & $\| \mathrm{A}$ & 9 & 11 & - \\
\hline & $\| \mathrm{B}$ & 8 & 9 & - \\
\hline & III & 14 & 2 & - \\
\hline
\end{tabular}




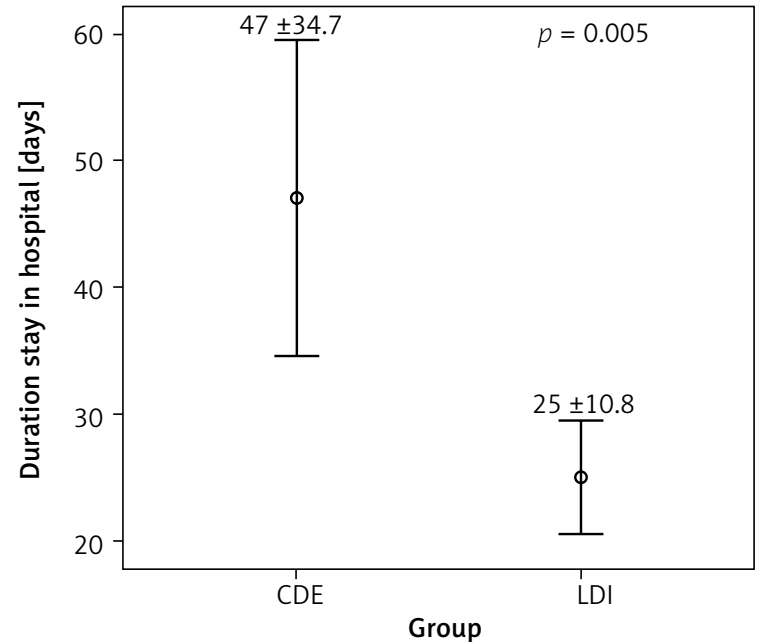

Figure 2. Length of patients' stay in hospital of the different groups

\section{Statistical analysis}

Statistical analysis was performed using SPSS (Statistical Package for Social Sciences) for Windows. Differences between means were evaluated using Student's $t$ test for parametric criteria and the $\chi^{2}$ test was used for nonparametric criteria. The Mann-Whitney test was used for small samples. A $p$ value of $<0.05$ was considered statistically significant.

\section{Results}

Fifty-seven patients were treated in the Department of Plastic and Reconstructive Surgery during 2 years. Thirty-two patients were in the CDE group and 25 patients were in the LDI group. Most patients were male (45 male vs. 12 female) and there was a significant difference $(p<0.001)$. The average age of the burned patients was $46.3 \pm 13.4$ years. The age of males and females was similar (female: 46.4 \pm 16.9 years vs. male: $46.3 \pm 12.5$ years; $p=0.11$ ). There was no significant difference comparing age and sex between the two groups. Thirty-eight patients $(66.7 \%)$ were smokers ( 35 males and 3 females). The main cause of burns was flame $-43 \mathrm{ca}$ ses $(75.4 \%)$. Thirty-three patients $(57.9 \%)$ had deep burns. The general patients' characteristics are shown in Table I.

LDI scan correlated with biopsy in 22/25 patients $(88 \%)$, while clinical investigation correlated with biopsy only in $23 / 32$ patients (71.9\%).

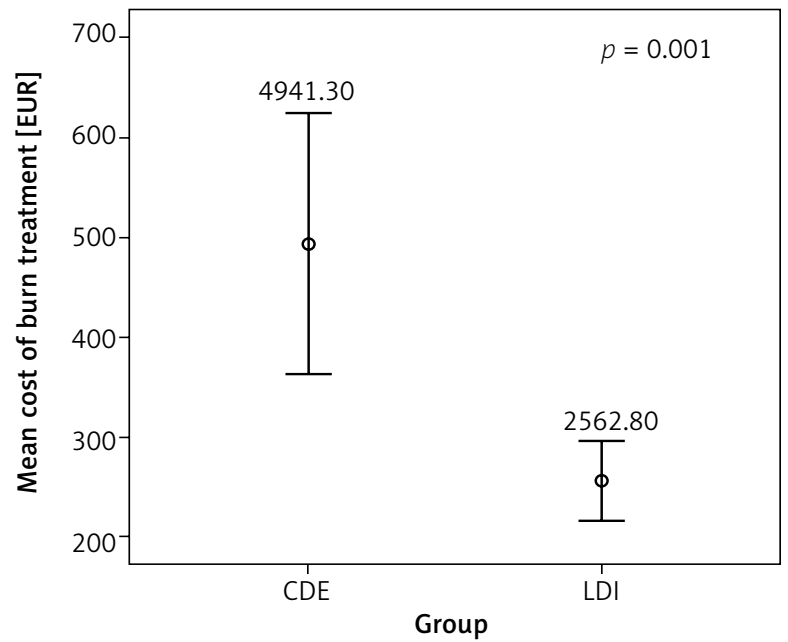

Figure 3. Cost of burn treatment of the different patient groups

The mean length of stay in hospital was significantly higher in the CDE group compared with the LDI group (Figure 2). The mean cost of treatment of burned patients was significantly more expensive in the CDE group (Figure 3). The patients from our database were divided into 4 subgroups: superficial and deep burn subgroups and conservative and surgical treatment subgroups. The length of stay in hospital and cost of treatment were analyzed in these subgroups and compared between different burn examination methods (CDE and LDI scan). The patients of the CDE group with deep burns and who underwent surgery had a significantly longer stay in hospital and received significantly more expensive treatment compared with patients of the LDI scan group (Table II).

The perfusion velocity of superficial burns was significantly higher compared with deep burns during the LDI scan at $72 \mathrm{~h}, 7^{\text {th }}$ and $14^{\text {th }}$ day after burns $(287.3$ $\pm 100.7 \mathrm{PU}$ vs. $162.3 \pm 120.9 \mathrm{PU}, p=0.003 ; 427.7 \pm 220.9$ PU vs. $120 \pm 52.4 \mathrm{PU}, p=0.004 ; 658.7 \pm 176.6 \mathrm{PU}$ vs. 313 $\pm 87.7 \mathrm{PU}, p=0.032$ ). Decrease of perfusion velocity of burned tissue during LDI scan investigation was the indication for surgery of burned patients (Figure 4).

\section{Discussion}

The relation between morbidity and mortality of burns and low socioeconomic status, profession, background and family status of patients has been proven by many social investigators $[1,8,9]$. Social 
Table II. Comparison of length of stay in hospital and cost of treatment of different subgroups using different burn wound examinations

\begin{tabular}{|lccc|}
\hline Subgroup & \multicolumn{2}{c}{ Group } & \multirow{2}{*}{ Value of $p$} \\
\cline { 2 - 3 } & CDE $(n=10)$ & LDI $(n=14)$ & NS \\
\hline Superficial burns (length of stay in hosp.) [days], $n=24$ & $19.1 \pm 12.3$ & $22.5 \pm 10.9$ & NS \\
\hline Superficial burns (cost of treatment) [EUR], $n=24$ & 2326.54 & 2045.48 & 0.001 \\
\hline Deep burns (length of stay in hosp.) [days], $n=33$ & CDE $(n=22)$ & LDI $(n=11)$ & 0.003 \\
\hline Deep burns (cost of treatment) [EUR], $n=33$ & $59.7 \pm 33.8$ & $28.3 \pm 10.2$ & NS \\
\hline & 6129.83 & 3221.15 & NS \\
\hline $\begin{array}{l}\text { Conservative treatment (length of stay in hospital) [days], } \\
n=20\end{array}$ & $26.8 \pm 11.5$ & $22.4 \pm 7.5$ & 2016.93 \\
\hline Conservative treatment (cost of treatment) [EUR], $n=20$ & 2539.43 & LDI $(n=12)$ & 0.012 \\
\hline & CDE $(n=24)$ & LDI $(n=13)$ & 0.01 \\
\hline Surgery (length of stay in hospital) [days], $n=37$ & $53.7 \pm 36.9$ & $27.5 \pm 12.9$ & 3066.63 \\
\hline Surgery (cost of treatment) [EUR], $n=37$ & 5741.92 & & \\
\hline
\end{tabular}

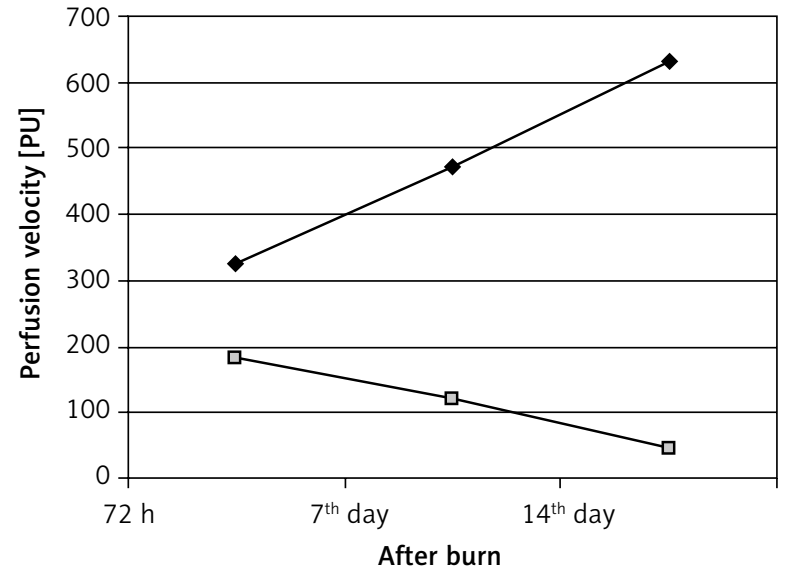

$\rightarrow$ - Deep burns

$\neg$ Superficial burns

Figure 4. Perfusion velocity of different burns during patients' follow-up

studies showed that those living in poverty, with low salary, had a high risk of burns. The elderly possessed higher risk too [1]. Males have more burn injuries than females but mortality of burns does not depend on sex. Males have more serious burn injuries compared to females $[2,9,10]$. We did not analyze the social or economic status of burn patients in our clinical study, but most of them were smokers and males. We had 43 patients of flame injuries and half of them were drowsing with a smoldering cigarette.

Early excisions of deep burn tissue reduce the length of stay and the cost of treatment, and decrease the risk of scar hypertrophy development [3-5, 7, 11]. Therefore it is important to assess and treat deep burns in the early period. Our clinical study proved that length of stay in hospital and cost of treatment of patients with deep burns were significantly different in the clinical burn depth examination group compared with the LDI group. There was no significant difference between the groups of patients with superficial burns comparing length of stay and cost of treatment.

Clinical burn depth examination is still popular in clinical practice [6, 7]. However, many clinical studies have shown that sensitivity of clinical burn depth examination varied from $50 \%$ to $72 \%$ compared to biopsy [3, 6, 11-13]. Burns of IIA degree can be assessed properly by means of clinical examination up to $66 \%$, IIB degree (when the burn wound heals in up to 2 weeks) up to $77 \%$, and IIB degree (when the burn wound heals in up to 3 weeks) only up to $50 \%$. The clinical burn depth examination is a subjective method and it depends on the experience of physicians $[6,14]$.

Biopsy is the "gold standard" to diagnose the depth of burn wounds. The sensitivity of biopsy can 
reach $100 \%[6,7,11,15,16]$. The burn wound biopsy is recommended to be performed within 48-72 $\mathrm{h}$ after injury, because the blood perfusion of burn tissue can change during this period and impair the results $[6,7]$. Biopsies of our clinical study were performed $72 \mathrm{~h}$ after injury, in order to prevent wrong results. The biopsy is an invasive technique and it can determine undesirable postoperative complications such as wound bleeding or suppuration [7]. Laser Doppler imaging scan is a non-invasive technique with sensitivity of $97 \%$. Laser Doppler imaging scan allows early diagnosis of deep burns and reduces the length of stay and cost of treatment [3, 7, 11, 12] Laser Doppler imaging scan did not correlate with biopsy of three patients from our study. The reason for this could be lubricants which were used during burn wounds dressing and were not cleaned carefully before the LDI scan. Deep burns opposite superficial burns were seen during the LDI scan. Clinical studies show that lubricants used for burn wound dressing reduce detection of blood perfusion during LDI investigation. The recommendation is to clean the burn wound carefully before the $\operatorname{LDI}$ scan $[4,11$, 17-19].

The LDI scan is a non-invasive, painless and easily performed method for burn depth evaluation. Jeng et al. [11] proved that the LDI scan helps to assess early superficial burns and prevent unnecessary surgery. There are many discussions between authors about the technique of LDI scan. Some of them propose $15-30 \mathrm{~cm}$ distance between the scanner and burn wound and a scan angle of $90^{\circ}[5,7,12,20]$. Other authors propose a distance of $50-70 \mathrm{~cm}$ and scan angle of $30-110^{\circ}[4,11,13,15]$. We decided to choose the first recommendation for our clinical study.

The blood perfusion is higher compared to normal tissue in the superficial burn tissue and considerably less in the deep burn tissue. The burn wound blood microcirculation becomes worse within 12-24h after the burn. The blood perfusion increases after $48 \mathrm{~h}$ and metabolic processes start in the burn wound $[4,12,20]$. Based on pathogenesis of burn tissue the recommendation to perform the LDI scan is $36-72 \mathrm{~h}$ after injury. The results can be false in the case of assessment of burn wound up to $24 \mathrm{~h}$ after injury [7]. In our clinical study we performed the first clinical burn depth examination and LDI scan $72 \mathrm{~h}$ after the burn.

According to Jeng et al. [11], if the perfusion velocity of the burn wound was 150-250 PU, the burn was deep and these patients should undergo surgery. If perfusion velocity was $>250$ PU the burn was superficial and these patients were referred for conservative treatment. However, successful results of conservative treatment were $>50 \%$ if perfusion velocity was > $250 \mathrm{PU}$ [19-21]. The perfusion velocity of burn wounds was measured repeatedly by other authors $[4,7,11]$. The results were slightly different: perfusion velocity 0-250 PU in the case of deep burns and patients were referred for surgery. If perfusion velocity was 250-625 PU, conservative treatment was performed with follow-up and repeated LDI scan later. If perfusion velocity decreased after repeated LDI scan, surgery was indicated. If perfusion velocity was > $625 \mathrm{PU}$, conservative treatment and spontaneous epithelization of burn wounds finally appeared $[4,7,11,13,16,20]$. We followed previous authors' recommendation for burned patients' indication for surgery in our clinical study. All patients of the LDI group with perfusion velocity less than 250 PU underwent surgery and patients were treated conservatively and followed up if perfusion velocity was > 250 PU.

However, the situation of patients with superficial (first degree burn) and deep (third degree burn) burns is certainly clear. The former are treated conservatively, while the latter undergo surgery. The most complicated situation is with IIA (superficial burn) and IIB (deep burn) degree burns. Sometimes IIA burns needs surgery and for patients with IIB burns enough conservative treatment and normal tissue epithelization is seen. It is necessary to determine a minimal perfusion velocity parameter during the LDI scan, which will enable one to choose the approach of treatment. We had 37 patients (20 patients with IIA degree burn and 17 patients with IIB degree of burn) with these degree burns in our study. Five patients of 20 with IIA degree burn were treated surgically and 4 patients of 17 with IIB degree burn were treated conservatively and normal tissue epithelization was seen. The mean perfusion velocity of these IIA patients was 264.80 PU and 305.50 PU of IIB patients. We did not calculate the prognostic parameter of perfusion velocity using a ROC curve, because we needed more cases of these types of patients.

\section{Conclusions}

Laser Doppler imaging scan is superior compared to clinical burn depth examination. Laser Doppler 
imaging is effective for assessment of deep burns in the early period of injury. The length of stay and the cost of treatment of burn patients depend on early diagnosis of deep burns and well-timed surgical treatment of burn wounds.

\section{References}

1. Park JO, Shin SD, Kim J, et al. Association between socioeconomic status and burn injury severity. Burns 2009; 35: 482-90.

2. Santaniello JM, Luchette FA, Esposito TJ, et al. Ten year experience of burn, trauma, and combined burn/trauma injuries comparing outcomes. J Trauma 2004; 57: 696-700.

3. Brown RF, Rice P, Bennett NJ. The use of laser Doppler imaging as an aid in clinical management decision making in the treatment of vesicant burns. Burns 1998; 24: 692-8.

4. Droog EJ, Steenbergen W, Sjoberg F. Measurement of depth of burns by laser Doppler perfusion imaging. Burns 2001; 27: 561-8.

5. Holland AJ, Martin HC, Cass DT. Laser Doppler imaging prediction of burn wound outcome in children. Burns 2002; 28: 11-7.

6. Monstrey S, Hoeksema H, Verbelen J, et al. Assessment of burn depth and burn wound healing potential. Burns 2008; 34: 761-9.

7. Stewart CJ, Frank R, Forrester KR, et al. A comparison of two laser-based methods for determination of burn scar perfusion: laser Doppler versus laser speckle imaging. Burns 2005; 31: 744-52.

8. Edelman LS. Social and economic factors associated with the risk of burn injury. Burns 2007; 33: 958-65.

9. Shin SD, Suh GJ, Sung J, et al. Epidemiologic characteristics of death by burn injury from 1991 to 2001 in Korea. Burns 2004; 30: 820-8.

10. Kobayashi K, Ikeda H, Higuchi R, et al. Epidemiological and outcome characteristics of major burns in Tokyo. Burns 2005; 31 Suppl. 1: S3-11.

11. Jeng JC, Bridgeman A, Shivnan L, et al. Laser Doppler imaging determines need for excision and grafting in advance of clinical judgment: a prospective blinded trial. Burns 2003; 29: 665-70.

12. Kloppenberg FW, Beerthuizen GI, ten Duis HJ. Perfusion of burn wounds assessed by laser doppler imaging is related to burn depth and healing time. Burns 2001; 27: 359-63.

13. La Hei ER, Holland AJ, Martin HC. Laser Doppler imaging of paediatric burns: burn wound outcome can be predicted independent of clinical examination. Burns 2006; 32: 550-3.

14. Johnson RM, Richard R. Partial-thickness burns: identification and management. Adv Skin Wound Care 2003; 16: 178-87.

15. Vo LT, Papworth GD, Delaney PM, et al. A study of vascular response to thermal injury on hairless mice by fibre optic confocal imaging, laser doppler flowmetry and conventional histology. Burns 1998; 24: 319-24.

16. Watts AM, Tyler MP, Perry ME, et al. Burn depth and its histolog ical measurement. Burns 2001; 27: 154-60.

17. Bray R, Forrester K, Leonard C, et al. Laser Doppler imaging of burn scars: a comparison of wavelength and scanning methods. Burns 2003; 29: 199-206.

18. Forrester KR, Tulip J, Leonard C, et al. A laser speckle imaging technique for measuring tissue perfusion. IEEE Trans Biomed Eng 2004; 51: 2074-84.
19. Kernick DP, Shore AC. Characteristics of laser Doppler perfusion imaging in vitro and in vivo. Physiol Meas 2000; 21: 333-40.

20. McGill DJ, Sorensen K, MacKay IR, et al. Assessment of burn depth: a prospective, blinded comparison of laser Doppler imaging and videomicroscopy. Burns 2007; 33: 833-42.

21. Allely RR, Van-Buendia LB, Jeng JC, et al. Laser Doppler imaging of cutaneous blood flow through transparent face masks: a necessary preamble to computer-controlled rapid prototyping fabrication with submillimeter precision. J Burn Care Res 2008; 29: $42-8$.

Received: 13.01.2013, accepted: 10.08.2013. 